\title{
ARTICLE
}

\section{Dementia in schizophrenia}

\author{
Raghavakurup Radhakrishnan, Robert Butler \& Laura Head
}

\begin{abstract}
Raghavakurup Radhakrishnan is an ST6 in old age psychiatry at St Clement's Hospital, Ipswich. His research interests include cognition and memory in dementia, depression, schizophrenia in late life and cognitive functioning after electroconvulsive therapy. Robert Butler is a consultant old age psychiatrist with Suffolk Mental Health Partnership NHS Trust, at St Clement's Hospital, Ipswich. His interests include dementia, depression in later life and service provision. Laura Head is a consultant old age psychiatrist with Suffolk Mental Health Partnership NHS Trust, working at Minsmere House, Ipswich. Her interests include crisis and in-patient care of older people with mental health problems, and medical management. Correspondence Dr Robert Butler, Consultant Old Age Psychiatrist, St Clement's Hospital, Foxhall Road, Ipswich IP3 8LS, UK. Email: robbutler100@hotmail.com
\end{abstract}

\begin{abstract}
SUMMARY
A growing body of evidence suggests that the most common type of dementia in schizophrenia differs from Alzheimer's disease in its clinical features, natural course, neuropathology, neuroanatomical substrates and prognosis. Furthermore, there is some evidence that the risk of developing cognitive impairment and its progression in earlyonset schizophrenia differ compared with late- or very-late-onset schizophrenia. The diagnosis and management of dementia in schizophrenia is challenging for both general adult and old age psychiatrists. This article reviews the evidence base regarding dementia in schizophrenia. It discusses the diagnosis of dementia in schizophrenia, its management and prognosis, and identifies some future research opportunities.
\end{abstract}

\section{DECLARATION OF INTEREST}

L. H. has undertaken commercial drug trials.

Cognitive impairment is a well-recognised and cardinal feature of schizophrenia (Keefe 2007). Given the biological effects of ageing on the brain, it is important to consider the cognitive manifestations of schizophrenia in the context of ageing. Significant issues include the natural history of cognitive dysfunction over the lifespan, its progression to dementia, and the temporal relationship between psychosis and cognitive deficits. In this context, it helps to have a precise definition of cognitive impairment, and we find the following useful: 'a level of cognitive functioning suggesting a consistent severe impairment and/ or a significant decline from premorbid levels considering patients' educational, familial and socioeconomic background' (Keefe 2007). Cognitive impairment has a trajectory over the course of schizophrenia that is different from that of psychotic symptoms and it may contribute to a poor functional outcome in older patients (Davidson 1995).

\section{Cognitive impairment in schizophrenia}

A number of meta-analyses have examined the extent of cognitive impairment in people with schizophrenia (Heinrichs 1998; Aleman 1999; Bokat 2003). Large effect sizes (between 1 and 1.5), representing robust evidence of impairment, have been reported across a multitude of cognitive domains, including current IQ, category fluency, verbal memory, abstract thinking, language, sustained attention and response inhibition. Symbol coding tasks yielded some of the most robust evidence, with very large effect sizes. However, Hyde et al (1994) performed a crosssectional study to assess cognitive performance in schizophrenia across a wide age range. A comparison of scores on cognitive measures recorded for five age-derived cohorts showed no evidence of accelerated decline on the MiniMental State Examination (MMSE), the Dementia Rating Scale, list learning, semantic fluency or perseverative errors on the Wisconsin Card Sorting Test.

\section{Do people with schizophrenia develop dementia?}

In his Lehrbuch der Psychiatrie of 1893, Kraepelin used the term 'dementia praecox' (premature dementia) for schizophrenia because of its onset in early life and the characteristic development of 'mental enfeeblement'. He emphasised the progressive deterioration of cognition but noted that memory was spared. He distinguished this condition from paraphrenia in which cognitive impairment did not occur.

Over a century later, there is uncertainty about the course of cognitive function with ageing in schizophrenia. Early studies of the progression of dementia over the lifespan in individuals with schizophrenia have shown inconsistent results, although they were limited by a lack of standardised diagnostic criteria and specific evaluation techniques.

In a study of long-term in-patients with schizophrenia who had survived into old age, Harvey et al (1999a) followed up a group for 30 months using the Clinical Dementia Rating (CDR). Over this period, $30 \%$ of the patients deteriorated, from a baseline of minimal or mild cognitive and functional impairment to impairments severe enough to warrant a secondary diagnosis of dementia.

Although many studies suggest a model of schizophrenia as a progressive dementing illness, others have supported the view that cognitive impairment in schizophrenia fits the model of a static encephalopathy (Goldberg 1993; Hyde 1994). 


\section{Evidence from international studies}

\section{Studies examining cognitive impairment in older people with schizophrenia}

Ciompi (1980) described evaluations of mostly elderly people surviving from a large sample of patients with schizophrenia. He estimated that $25 \%$ had moderate to severe cognitive deficits, including memory disturbance and disorientation. This study of chronically institutionalised older patients demonstrated the presence of agerelated impaired global cognitive performance as measured by the MMSE. Other researchers have estimated that more than $80 \%$ of older people with schizophrenia show significant cognitive impairment (Keefe 2007).

\section{Studies showing a significant decline in cognition over time}

In a re-evaluation of the IOWA 500 study of a cohort of people with hebephrenic or catatonic schizophrenia followed up for 40 years, Winokur et al (1987) found that the subgroup of older participants was significantly more likely to have worsened orientation and memory.

In a follow-up of older patients with schizophrenia after discharge from long-term care in psychiatric hospital, Harvey et al (1999b) noted an average reduction of 3.8 points on the MMSE over a period of 2.5 years. Friedman et al (2001) evaluated the cognitive and functional status of people with schizophrenia aged between 20 and 80 years in a 6 -year follow-up study, and compared the subgroup of patients aged 50 and over with healthy individuals and patients with Alzheimer's disease, also aged 50 and over. They measured cognitive and functional decline using CDR scores. They found a significant age-group effect on the magnitude of cognitive decline for participants with schizophrenia. For those under the age of 65, the proportional risk of cognitive and functional decline in schizophrenia was negligible. However, the proportional risk rose from $38 \%$ for those aged $65-70$, to $75 \%$ for the 70-75 age group and reached $100 \%$ for those aged $75-80$. Neither prior leucotomy, electroconvulsive therapy (ECT) or insulin coma therapy, nor current exposure to antipsychotics showed adverse effects on current cognitive functioning. The limitations of this study included the use of relatively non-specific measures (MMSE and CDR) and the fact that the patients had been institutionalised for long periods.

\section{Studies showing a lesser decline in cognition}

An early study of older in-patients with schizophrenia (Harvey 1995) demonstrated that cognitive changes were very slow over time, despite low MMSE scores at initial assessment. The authors concluded that cognitive functioning in older people with schizophrenia is different from that seen in Alzheimer's disease.

Heaton et al (1994) compared three groups of people with schizophrenia (young people with early-onset schizophrenia, older people with earlyonset disorder, and people with late-onset, i.e. after age 45 , disorder) with ambulatory patients with Alzheimer's disease and normal controls. They concluded that cognitive impairment in schizophrenia is unrelated to current age, age at onset or duration of illness. They also suggested that there is an encephalopathy associated with schizophrenia that is non-progressive and that has a different pattern of deficits from that seen in Alzheimer's disease.

\section{The risk of dementia in late-onset and very-late- onset schizophrenia}

Late-onset schizophrenia is usually defined as onset after 40 years of age, and very-late-onset as after 60 years of age. Studies of schizophrenia of late and very late onset are few and inconsistent in their results.

Palmer et al (2003) compared changes in cognition over 1 and 2 years for out-patients with late-onset schizophrenia-spectrum disorders, earlier-onset schizophrenia-spectrum disorders, Alzheimer's disease with psychosis, or Alzheimer's disease with baseline MMSE scores $>25$, and healthy comparison participants. Cognitive changes among participants in the two schizophrenia-spectrum disorder groups were similar to those in the healthy controls, whereas both Alzheimer's disease groups showed greater declines in cognition. The authors concluded that late-onset schizophrenia is a neurodegenerative disorder and, in agreement with earlier studies, a static encephalopathy.

In another study (Laks 2006), involving a small number of Brazilian patients with late-onset schizophrenia, none of the participants developed observable dementia during 1-year follow-up. Cognition and activities of daily living remained stable over the course of the year. However, a longer-term Australian follow-up study, also with a small sample size, produced very different results. Brodaty et al (2003) compared patients with onset of schizophrenia at the age of 50 years or over, but without dementia, with a healthy control group. All participants were assessed at baseline, after 1 year and after 5 years on measures of psychopathology, cognition and general functioning. At the 5-year follow-up, $47 \%$ of participants in the schizophrenia group met the criteria for dementia, for which the 
most common diagnosis was Alzheimer's disease. None of the control group had dementia.

In a case-register study in Denmark, Kørner et al (2009) followed up a very large sample of first-contact patients with late- and very-late-onset schizophrenia for between 3 and 4.6 years. They compared these individuals with two age-matched control groups: patients with osteoarthritis and a sample of the general population. After 3-4.6 years, the risk of receiving a diagnosis of dementia was two to three times higher for the schizophrenia group than for the control groups. Among the participants who developed dementia during follow-up, the most common diagnosis in the schizophrenia group was unspecified dementia, whereas in the general population and osteoarthritis groups it was Alzheimer's disease.

This Danish study suggests that, although people with late-onset and very-late-onset schizophrenia have an increased risk of developing dementia, it does not seem to be due to an increased risk of developing Alzheimer's disease, thus pointing to the possibility that they are developing a different type of dementia.

Box 1 summarises the evidence from international studies.

\section{Neuropathology and post-mortem evidence}

Kørner et al's (2009) finding of an increased risk of developing dementia in their schizophrenia cohort might be explained by confounding diagnostic factors. For example, Alzheimer's disease or vascular dementia might have presented atypically or have been undiagnosed by clinicians reluctant to make a diagnosis in the context of schizophrenia. People with schizophrenia often have unhealthy lifestyles and inadequate physical healthcare, both of which are vascular risk factors for Alzheimer's disease and vascular dementia. However, post-mortem neuropathological studies do not support this hypothesis.

B0X 1 Cognitive impairment in schizophrenia

- Cognitive impairment is a feature of schizophrenia

- Over $25 \%$ of older people with schizophrenia have moderate to severe cognitive impairment

- Most studies have found a significant decline in cognition over time in schizophrenia

- Conversely, some studies have found cognitive impairment to be relatively stable in schizophrenia

- The proportion of people who develop dementia in later life among those with schizophrenia appears to be higher than in the general population
B0X 2 Post-mortem evidence

- Most people with schizophrenia and moderate to severe cognitive impairment do not have Alzheimer's disease pathology

- Post-mortem studies have generally not found an increased incidence of Alzheimer's disease in people with schizophrenia

Purohit et al (1993) studied the presence of senile plaques and neurofibrillary tangles in the post-mortem brains of a small number of elderly patients with schizophrenia and cognitive impairment, comparing them with the brains of age-matched patients with Alzheimer's disease. It was noted that none of the brains from the group with schizophrenia showed a sufficient degree of senile plaques and neurofibrillary tangles to make the diagnosis of Alzheimer's disease. Despite advanced age and cognitive impairment, however, the density of senile plaques was sparse in the majority of samples from the schizophrenia group. The authors concluded that cognitive impairment cannot be explained by increased vulnerability to Alzheimer's disease. Other (mostly smallsample) studies using immunohistochemistry have failed to show any increased incidence of Alzheimer's disease pathology in people dying with schizophrenia and cognitive impairment.

A subsequent study by Purohit et al (1998) examined 100 consecutive autopsy brains from patients with schizophrenia, and compared them with the brains from normal controls and from patients with non-schizophrenic psychoses. In previous cognitive assessments, $72 \%$ of the patients with schizophrenia had shown moderate to severe cognitive impairment. However, postmortem examination revealed that only $9 \%$ met neuropathological criteria for Alzheimer's disease. This study demonstrates that, although severe cognitive impairment is commonly observed in schizophrenia, it is only occasionally due to comorbid Alzheimer's disease.

The findings from post-mortem studies are summarised in Box 2.

If the pathology is not that of Alzheimer's disease, what is its neuropathological basis? Most dementia in schizophrenia is not characterised by classic, histologically identifiable neuropathology and most is probably not the result of an identifiable dementing illness.

\section{Neurochemistry}

A number of neurochemical abnormalities have been identified in cognitively impaired patients 
with schizophrenia. Although some of these are shared with Alzheimer's disease, many are not. For example, cortical somatostatin and serotonin levels are similar in schizophrenia and Alzheimer's disease, whereas cortical corticotrophinregulating hormone and acetylcholine levels are dissimilar. Cognitively impaired patients with schizophrenia show cortical deficits of vasoactive intestinal peptide and neuropeptide $\mathrm{Y}$, and increased senile plaque concentrations in the cingulate and temporal cortex (Powchik 1998). In addition, there is some evidence of a reduction in concentrations of serotonin and noradrenaline in cognitive impairment. It has also been shown that people with a diagnosis of schizophrenia and dementia without Alzheimer's disease do not show significantly elevated levels of amyloid $\beta$-peptide, which is increased in the post-mortem brains of Alzheimer's disease patients (Religa 2003). This last study suggested that antipsychotics may actually protect against the development of Alzheimer's disease pathology.

\section{Diagnosis}

The assessment of people with schizophrenia presenting with features of dementia can be difficult for several reasons. First of all, it can be clearly demonstrated that a substantial percentage of people with schizophrenia develop cognitive dysfunction early in their illness, so a retrospective history may not give a clear-cut picture of recent changes. However, a detailed history from both the patient and an informant with close personal knowledge of them over time may reveal a pattern of increasing cognitive decline. The history and assessment should include orientation, memory, personality changes and activities of daily living. Effective cognitive testing is essential on presentation, as is information about the patient's previous level of functioning.

Diagnosis at this stage would be made easier if cognitive testing early in the lifespan of patients with schizophrenia were routine. There is a growing consensus that cognitive deficits characterising schizophrenia should be incorporated into the major diagnostic systems, including the DSM and ICD (Bora 2010). Such a development would prompt clinicians to assess a patient's cognitive profile early in the course of schizophrenia. This, in turn, might stimulate new treatment methods and promote better management of cognitive impairment and dementia in schizophrenia. The emphasis should be on recognising whether cognitive deficits have been stable over time or there has been a significant decline from a premorbid level of cognitive function, particularly

\section{B0X 3 Clinical diagnosis}

- Always assess cognition in people with schizophrenia

- From the history, establish the pattern of cognitive impairment and level of functioning over time

- Establish whether cognitive impairment fluctuates with psychotic symptoms (less likely to be a dementia) or shows a slow, steady decline (more likely to be a dementia)

in later life. The question of whether longitudinal assessments of cognitive function would have predictive value needs further research.

The correct diagnosis of dementia in people with schizophrenia (Box 3) is largely dependent on the expertise and skill of the clinician carrying out the assessment. However, the measurement of cognitive function required to inform the diagnosis raises a vexing question: What tools, measures and approaches should the clinician use to assess cognition? To complicate matters further, cognitive decline, a core feature of both schizophrenia and dementia, is found to some extent in normal ageing. Moreover, all these evaluations require the patient's cooperation.

\section{Assessment of cognitive function}

Many studies have shown that the MMSE (Folstein 1975) is not adequate to pick up cognitive deficits in complex disorders such as schizophrenia. There is evidence that the MMSE scores of people with schizophrenia remain relatively stable over time (Harvey 1995; Brodaty 2003; Palmer 2003). It will therefore take a long time for changes in cognitive function to become apparent using this measure.

The MATRICS Consensus Cognitive Battery (MCCB) assesses seven putatively separable domains of cognitive dysfunction in schizophrenia (Kern 2008; Nuechterlein 2008). In an ideal world, this tool could be used with every patient. However, practical and economic constraints facing many clinics and treatment settings make this unfeasible. Studies have shown that impairments on digit-symbol substitution tasks have the largest effect sizes among many different measures for characterising cognitive impairment in schizophrenia.

Another consideration is whether it is necessary to carry out a formal assessment of cognition, given that many clinicians will not have any experience in administering such measures. Scales such as the Schizophrenia Cognition Rating Scale (Keefe 2006) may be easier for clinicians to administer as they are similar in format to a traditional diagnostic assessment. 
Tools designed for use in Alzheimer's disease may be useful for the assessment of cognitive deterioration in schizophrenia. These include the Consortium to Establish a Registry for Alzheimer's Disease (CERAD) neuropsychological battery (Morris 1989) and the cognitive subscale of the Alzheimer's Disease Assessment Scale Late version (ADAS-L Cog). The CERAD battery is designed to measure all aspects of cognitive functioning, whereas the ADAS-L Cog is specific to late-stage Alzheimer's dementia.

A study involving a sample of elderly patients with schizophrenia (Bowie 2002) concluded that the ADAS-L Cog reliably and validly assesses cognition in the presence of severe cognitive impairment. The authors felt that the CERAD battery was not adequate in a particularly lowfunctioning population.

Zakzanis et al (2003) conducted neuropsychological assessments to identify the best tests to distinguish between Alzheimer's disease and late-onset schizophrenia. Results showed that the similarities subtest of the Wechsler Adult Intelligence Scale - Revised (Wechsler 1981) and the California Verbal Learning Test (Delis 1987) are particularly suitable.

The Milan Overall Dementia Assessment (MODA; Brazzelli 1994) has also been used to assess cognitive impairment in people with schizophrenia (Lepore 2009). It was found to be a valid tool for identifying cases of dementia in this group, and results were not influenced by an individual's age or level of education. The authors recommended that the MODA might be useful in routine clinical practice.

The use of such tools (Box 4) in the assessment of patients is more time-consuming than routine clinical evaluation. Consequently, it may not be

BOX 4 Cognitive testing

- Assess a broad range of cognition, including memory (short- and long-term), orientation, concentration, language, praxis and executive function

- The MMSE has limitations but is better than using no scale

- Slightly longer scales such as the ADAS-L Cog and Addenbrooke's Cognitive Examination - Revised (ACE-R; Mioshi 2006)) give more useful information

- There are specific tools for dementia and schizophrenia, such as the MATRICS

- There are specific tools for Alzheimer's disease, such as the CERAD

- There are specific tools for the negative features of schizophrenia, such as the PANSS feasible to use scales in every case. It may be more appropriate to take a detailed history and carry out a brief cognitive assessment in all cases and to reserve specific tools for patients with difficult presentations.

\section{Assessment of social and occupational functioning}

Both DSM-IV and ICD-10 diagnostic criteria for dementia include impaired social and occupational functioning. It is therefore necessary to establish a decline in social functioning to make a diagnosis of dementia. However, such impairment may also occur as negative symptoms of schizophrenia, and the differentiation of negative symptoms from a functional decline needs careful assessment. The Positive and Negative Syndrome Scale (PANSS; Kay 1987) may be useful for the identification of negative symptoms. The Social Adaptive Functioning Evaluation (SAFE) scale (Harvey 1997) is a 17-item scale that measures socialinterpersonal, instrumental and impulse-control deficits and it has a high interrater reliability. These tools may be particularly useful for patient follow-up and for developing treatment strategies.

\section{Brain imaging and diagnosis}

Technological advances have led to a greater use of structural and functional brain imaging to assist with the diagnosis of dementia in people with evidence of cognitive decline. Additional information obtained from these investigations helps clinicians to make an accurate diagnosis and formulate a treatment plan. In clinical practice, structural magnetic resonance imaging (MRI) and computed tomography (CT) scanning are widely used, and radiologists interpret results on the basis of visual readings.

Structural imaging is often carried out to identify potentially treatable causes of dementia, such as normal pressure hydrocephalus or spaceoccupying lesions, or to clarify the diagnosis by detecting cerebral infarctions (strokes) or patterns of atrophy. In cognitively impaired patients without a definite clinical diagnosis, MRI may be helpful in reaching a diagnosis. Without MRI investigation the clinical diagnosis of vascular dementia may be unreliable. Image analysis programs that quantify regional volumes in MRI (and CT) have shown that measures of medial temporal or hippocampal atrophy can distinguish people with Alzheimer's disease from controls. Several studies have shown ventricular enlargement, cerebral infarction or white matter ischaemic change using CT or MRI scans. However, these findings are non-specific in both Alzheimer's disease and vascular dementia. 
The results of CT investigation of brain structure in schizophrenia have been variable. The most consistent finding has been lateral ventricular enlargement, although the cause of this remains unclear. Computed tomography and MRI could give an insight into biological abnormalities in patients with schizophrenia and dementing illness, but studies are limited in this group. Förstl et al (1994) compared CT scans of patients with lateonset paranoid psychosis with those of patients with Alzheimer's disease and of elderly controls with dementia. They found that the ventricles and anterior and Sylvian fissures of the psychosis group were larger than in the controls but smaller than in those with Alzheimer's dementia. However, these non-specific findings alone do not help the clinician to distinguish dementia in schizophrenia from dementia due to Alzheimer's disease or vascular disease or, indeed, to distinguish people with dementia from those without.

Functional imaging technologies such as singlephoton emission computed tomography (SPECT), positron emission tomography (PET) and functional MRI (fMRI) also provide information about brain structure, but their spatial resolution is lower than with CT or structural MRI. The added value of functional scanning is the information provided on cerebral blood flow or glucose metabolism, even when structural deficits are not present. This can help the physician in the diagnosis and differential diagnosis of dementia (Silverman 2001). Patients with Alzheimer's disease typically show parietal, temporal, posterior cingulate and frontal deficits, whereas those with frontotemporal dementia show frontal and temporal deficits (Silverman 2001). Dementia with Lewy bodies has a pattern similar to that of Alzheimer's disease, but with additional occipital deficits. However, there are no studies of dementia associated with schizophrenia.

As the clinical use of neuroimaging techniques expands, investigators are exploring novel applications of existing techniques and developing others that measure various biological processes relevant to neurodegeneration. Combining neuroimaging studies with other procedures that provide data on genetic risk and biomarker measures from other tissues (e.g. blood serum, cerebrospinal fluid) might increase diagnostic sensitivity and specificity, as well as the predictive value of the information.

For a summary of this section, see Box 5 .

\section{Management}

A major issue in the management of dementia in schizophrenia is which services are best suited to provide care for patients (Box 6). Health policy

\section{B0X 5 Brain imaging}

- If the clinical assessment suggests an organic cause of cognitive decline, CT and MRI are useful for excluding normal pressure hydrocephalus, tumour and subdural haematoma

- CT and MRI changes are non-specific in schizophrenia; ventricular enlargement is the most common finding

- CT and MRI changes are also non-specific in Alzheimer's disease and vascular dementia; atrophy and small-vessel changes respectively are the most common findings, but these also occur in healthy older people

- Little is known about functional imaging (SPECT, PET or $\mathrm{fMRI}$ ) in this area.

in the UK requires the early identification and management of dementia and the active inclusion of people with dementia in the decision-making process (Department of Health 2001). However, there are only a few high-quality evaluations of models of care in dementia (National Collaborating Centre for Mental Health 2011), and even these show a particular lack of data from user and carer perspectives.

The Royal College of Psychiatrists (2006) has made recommendations for service provision for younger people with dementia. A study comparing old age psychiatrists and neurologists found that younger patients may be underinvestigated if solely managed by old age psychiatrists, and may not receive adequate follow-up if managed by neurologists (Cordery 2002). On the whole, old age psychiatrists may be best equipped to manage these patients, provided that they work in close collaboration with the appropriate specialists, for example neurologists.

\section{B0X 6 Management}

- General adult mental health services have expertise at managing schizophrenia, whereas older people's services specialise in managing dementia

- Memory clinics do not usually have the expertise to manage people with schizophrenia and cognitive impairment

- Antipsychotics usually improve cognition if the impairment is associated with psychotic symptoms

- Antipsychotics are contraindicated in people with primary dementia and non-cognitive symptoms of dementia, because of the increased risk of strokes and death

- Acetylcholinesterase inhibitors may be helpful, but are still being evaluated 
Memory clinics have become established as a response to an increased demand for dementia assessment and care. Such clinics do not adhere to a single type of service model or delivery, but they have features in common: they are usually for people with mild to moderate dementia; a thorough assessment is conducted; diagnoses are made; and pharmacological treatment may be initiated and monitored. Although it is possible to evaluate cases of dementia in schizophrenia in these clinics, an already overburdened service may not be able to meet all the demands of patients with such complex needs. Where dementia services are provided across the age range, they should construct themselves to be acceptable and to meet the needs of people with early-onset dementia and their carers as well as those with dementia in old age (Willis 2009). Services that are designed to treat people with dementia need to develop strategies to care for those with subjective memory impairment but no objective deficits.

People with comorbid schizophrenia and dementia may need specific management of their psychosis. It may be argued that general adult mental health services are better equipped to deal with schizophrenia, whereas old age mental health services have expertise in dementia. General adult and old age mental health services may need to cooperate to provide a service that meets all the needs of these patients. Both services should be able to advise people on healthy living (Box 7).

\section{Drug treatment}

\section{Antipsychotics}

Pharmacological management of schizophrenia has been available for many decades, and antipsychotics remain the mainstay of the treatment of schizophrenia in the elderly population. However, attempts to find out whether these treatments improve cognitive function in people with schizophrenia have shown mixed results.

The second-generation (atypical) antipsychotics may enhance memory and attention compared

B0X 7 Healthy living

- People with schizophrenia often have poor physical health associated with unhealthy lifestyles

- People with schizophrenia with or without dementia should be encouraged to live healthily

- Weight control, blood pressure control, good diet exercise, not smoking and avoiding diabetes may reduce the risk of developing dementia with first-generation agents (Keefe 1999), although their effect falls far short of restoring full cognitive function (Keefe 2004; Harvey 2005). Several metaanalyses of drug treatment in schizophrenia have suggested that second-generation antipsychotics can significantly improve cognition at first, but subsequent improvements with long-term treatment are usually modest (Keefe 1999). There is only limited evidence that long-term treatment can prevent the development of dementia in schizophrenia, nor do antipsychotics appear significantly helpful in its management.

\section{Acetylcholinesterase inhibitors}

Some evidence suggests that alterations in the central cholinergic system seen in people with schizophrenia, such as reduced numbers of muscarinic and nicotinic receptors in the cortex and hippocampus, may contribute to the cognitive deficits seen in the disorder (Friedman 2004). The acetylcholinesterase inhibitors donepezil, galantamine and rivastigmine are effective in dementia, especially mild to moderate Alzheimer's disease (Holden 2002; Raina 2008). However, trials using these medications for cognitive enhancement in schizophrenia have shown mixed results (Stip 2007; Buchanan 2008).

Few studies have described the use of acetylcholinesterase inhibitors in dementia associated with schizophrenia. In one, the possible beneficial effect of donepezil augmentation for the management of comorbid schizophrenia and dementia was studied by Stryjer et al (2003). Six people with chronic schizophrenia and comorbid dementia received donepezil (5 mg/day) in single-blind fashion as augmentation to their standard antipsychotic medication for a 4 -week period. A significant improvement was noted in MMSE scores and Clinical Global Impression (CGI) scores. In addition, three participants demonstrated improvement on the PANSS. The authors concluded that donepezil appears to be an effective treatment for the management of symptoms of dementia in people with comorbid schizophrenia and dementia.

In another study, 13 people with comorbid chronic schizophrenia and dementia received augmentative open-label oral rivastigmine (9 mg/day) for 12 weeks (Mendelsohn 2004). Improvements were recorded in aspects of cognitive function, activities of daily living and overall psychotic features.

Both of these studies involved small samples of patients and were open-label trials, and they require replication using larger, randomised double-blind designs. 


\section{Summary}

It is difficult to suggest a particular medication for patients with comorbid chronic schizophrenia and dementia on the basis of current evidence.

\section{Prognosis of dementia in schizophrenia}

Dementia shortens the lifespan. At any given age, people who have dementia are two to four times more likely to die than those who do not have the disease (Dewey 2001; Guehne 2006). The median survival time of people with dementia has been investigated in cohort studies and case series in various settings, and it ranges from 3 to 9 years (Dewey 2001; Xie 2008). However, longitudinal comparisons of the course of cognitive and functional decline have been very few in the subpopulation with dementia in schizophrenia. Mayer (1921) found that many of Kraepelin's patients with paraphrenic psychoses showed unequivocal signs of dementia later in the course of illness. Holden (1987) observed that people with late paraphrenia had significantly worse outcomes when they developed dementia.

As mentioned earlier, Goldberg et al (1993) stated that schizophrenia can be regarded as a static encephalopathy. Some studies suggest that the progressive cognitive decline experienced by people with poor-outcome (long-term institutionalised) schizophrenia is not a linear process across the lifespan, and the rate of decline may accelerate after the age of 65 (Friedman 2001).

Again, more studies are needed, on both the survival rates and prognosis of people with schizophrenia once they develop dementia (Box 8).

\section{The future}

The lack of evidence-based knowledge in the area of dementia in schizophrenia is highly evident. In particular, it is not yet clear whether there is an excess of dementia in schizophrenia, be it of early or late onset. A sustained cognitive impairment suggesting a diagnosis of dementia does not always occur, but Kraepelin's concept of dementia praecox appears to be right at least some of the time. It is debatable whether we under- or overdiagnose dementia in this group of patients. We need naturalistic studies to investigate this. Research needs to concentrate on accurately diagnosing dementia in schizophrenia using the best neuropsychological methods available, in combination with brain imaging and post-mortem examination. As we learn more about the genetics of schizophrenia and the subtypes of dementia, a complicated but better understood picture will emerge.

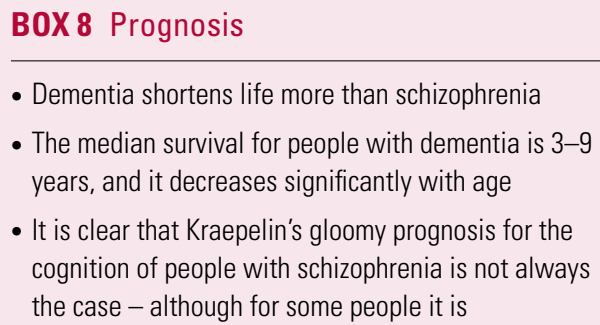

Another underresearched area is how best to manage dementia in schizophrenia. Healthy lifestyles are clearly desirable, but as yet there is little hard evidence of any benefit for people with schizophrenia or with dementia, let alone with both. Acetylcholinesterase inhibitors look promising as an augmentative treatment and there are many more antidementia medications under development.

As populations across the world age, so does the population of people with schizophrenia. Age is the biggest risk factor for dementia, and since over $20 \%$ of the general population of over-80-year-olds have dementia (Department of Health 2001), the proportion is likely to be higher among those with schizophrenia. We need to push the needs of this group up the health agenda and develop more and better services to meet them.

\section{References}

Aleman A, Hijiman R, De Haan EHF, et al (1999) Memory impairment in schizophrenia: a meta-analysis. American Journal of Psychiatry 156: 1358-66.

Bokat CE, Goldberg TE (2003) Letter and category fluency in schizophrenic patients: a meta-analysis. Schizophrenia Research 64: 73-8.

Bora E, Yucel M, Pantelis C (2010) Cognitive impairment in schizophrenia and affective psychoses: implications for DSM-V criteria and beyond. Schizophrenia Bulletin 36: 36-42.

Bowie CR, Harvey PD, Moriarty PJ, et al (2002) Cognitive assessment of geriatric schizophrenic patients with severe impairment. Archives of Clinical Neuropsychology 17: 611-23.

Brazzelli M, Capitani E, Della Sala S, et al (1994) A neuropsychological instrument adding to the description of patients with suspected cortical dementia: the Milan overall dementia assessment. Journal of Neurology, Neurosurgery and Psychiatry 57: 1510-7.

Brodaty H, Sachdev P, Koschera A, et al (2003) Long-term outcome of lateonset schizophrenia: 5-year follow-up study. British Journal of Psychiatry 183: 213-9.

Buchanan RW, Conley RR, Dickinson D, et al (2008) Galantamine for the treatment of cognitive impairments in people with schizophrenia. American Journal of Psychiatry 165: 82-9.

Ciompi $L$ (1980) Catamnestic long-term study on the course of life and aging of schizophrenics. Schizophrenia Bulletin 6: 606-18.

Cordery R, Harvey R, Frost C, et al (2002) National survey to assess current practices in the diagnosis and management of young people with dementia. International Journal of Geriatric Psychiatry 17: 124-7.

Davidson M, Harvey PD, Powchik P, et al (1995) Severity of symptoms in chronically institutionalized geriatric schizophrenic patients. American Journal of Psychiatry 152: 197-207. 


MCQ answers
$1 \mathrm{a} \quad 2 \mathrm{a} \quad 3 \mathrm{e} \quad 4 \mathrm{a} \quad 5 \mathrm{~d}$

Delis DC, Kramer JH, Kaplan E, et al (1987) California Verbal Learning Test Manual (Research Edition). Psychological Corporation/Harcourt Brace.

Department of Health (2001) National Service Framework for Older People. Department of Health.

Dewey ME, Saz P (2001) Dementia, cognitive impairment and mortality in persons aged 65 and over living in the community: a systematic review of the literature. International Journal of Geriatric Psychiatry 16: 751-61.

Folstein MF, Folstein SE, McHugh PR (1975) 'Mini-mental state': a practical method for grading the cognitive state of patients for the clinician. Journal of Psychiatric Research 12: 189-98.

Förstl H, Dalgalarrondo P, Riecher-Rössler A, et al (1994) Organic factors and the clinical features of late paranoid psychosis: a comparison with Alzheimer's disease and normal ageing. Acta Psychiatrica Scandinavica 89: $335-40$

Friedman JI, Harvey PD, Coleman T, et al (2001) Six-year follow-up study of cognitive and functional status across lifespan in schizophrenia: a comparison with Alzheimer's disease and normal aging. American Journal of Psychiatry 158: 1441-8.

Friedman JI (2004) Cholinergic targets for cognitive enhancement in schizophrenia: focus on cholinesterase inhibitors and muscarinic agonists. Psychopharmacology 174: 45-53.

Goldberg TE, Hyde TM, Kleinman JE, et al (1993) Course of schizophrenia: neuropsychological evidence for a static encephalopathy. Schizophrenia Bulletin 19: 797-804.

Guehne U, Angermeyer MC, Riedel-Heller S (2006) Is mortality increased in mildly cognitively impaired individuals? A systematic literature review. Dementia and Geriatric Cognitive Disorders 21: 403-10.

Harvey PD, White L, Parrella M, et al (1995) The longitudinal stability of cognitive impairment in schizophrenia. Mini-mental state scores at one- and two-year follow-ups in geriatric in-patients. British Journal of Psychiatry 166: 630-3

Harvey PD, Davidson M, Mueser KT, et al (1997) Social-Adaptive Functioning Evaluation (SAFE): a rating scale for geriatric psychiatric patients. Schizophrenia Bulletin 23: 131-45.

Harvey PD, Parella M, White L, et al (1999a) Convergence of cognitive and adaptive decline in late life schizophrenia. Schizophrenia Research 45: $32-40$.

Harvey PD, Silverman JM, Mohs RC, et al (1999b) Cognitive decline in late life schizophrenia: a longitudinal study of geriatric chronically hospitalized patients. Biological Psychiatry 45: 32-40.

Harvey PD, Rabinowitz J, Eerdekens M, et al (2005) Treatment of cognitive impairment in early psychosis: a comparison of risperidone and haloperidol in a large long-term trial. American Journal of Psychiatry 162 . 1888-95.

Heaton RK, Paulsen J, McAdams LA, et al (1994) Neuropsychological deficits in schizophrenics: relationship to age, chronicity, and dementia. Archives of General Psychiatry 51: 469-76.

Heinrichs RW, Zakzanis KK (1998) Neurocognitive deficits in schizophrenia: a quantitative review of the evidence. Neuropsychology 12: 426-45.

Holden NL (1987) Late paraphrenia or the paraphrenias? A descriptive study with a 10-year follow-up. British Journal of Psychiatry 150: 635-9.

Holden M, Kelly C (2002) Use of cholinesterase inhibitors in dementia. Advances in Psychiatric Treatment 8: 89-96.

Hyde TM, Nawroz S, Goldberg TE, et al (1994) Is there cognitive decline in schizophrenia? A cross-sectional study. British Journal of Psychiatry 164: $494-500$

Kay SR, Fiszbein A, Opler LA (1987) The Positive and Negative Syndrome Scale (PANSS) for schizophrenia. Schizophrenia Bulletin 13: 261-76.

Keefe R, Silva S, Perkins D, et al (1999) The effects of atypical antipsychotic drugs on neurocognitive impairment in schizophrenia: a review and meta-analysis. Schizophrenia Bulletin 25: 201-22.

Keefe RS, Seidman LJ, Christensen BK, et al (2004) Comparative effect of atypical and conventional antipsychotic drugs on neurocognition in firstepisode psychosis: a randomized, double-blind trial of olanzapine versus low doses of haloperidol. American Journal of Psychiatry 161: 985-95.
Keefe RSE, Poe M, Walker TM, et al (2006) The Schizophrenia Cognition Rating Scale: an interview-based assessment and its relationship to cognition, real-world functioning, and functional capacity. American Journal of Psychiatry 163: 426-32.

Keefe RSE, Fenton WS (2007) How should DSM-V criteria for schizophrenia include cognitive impairment? Schizophrenia Bulletin 33: 912-20.

Kern RS, Nuechterlein KH, Green MF, et al (2008) The MATRICS Consensus Cognitive Battery, Part 2: co-norming and standardization. American Journal of Psychiatry 165: 214-20.

Kørner A, Lopez AG, Lauritzen L, et al (2009) Late and very-late firstcontact schizophrenia and the risk of dementia - a nationwide register based study. International Journal of Geriatric Psychiatry 24: 61-7.

Laks J, Fontenelle LF, Chalita A, et al (2006) Absence of dementia in late-onset schizophrenia: a one year follow-up of a Brazilian case series. Arquivos de Neuro-psiquiatria 64: 946-9.

Lepore A, Borelli A, Patella RM, et al (2009) Cognitive impairment in patients affected by schizophrenia measured with MODA rating scale. Clinical Neuropsychiatry 6: 117-23.

Mayer W (1921) Über paraphrene Psychosen [On paraphrenic psychoses]. Zeitschrift für die Gesamte Neurologie und Psychiatrie 71: 187-206.

Mendelsohn E, Rosenthal M, Bohiri Y, et al (2004) Rivastigmine augmentation in the management of chronic schizophrenia with comorbid dementia: an open-label study investigating effects on cognition, behaviour and activities of daily living. International Clinical Psychopharmacology 19: 319-24.

Mioshi E, Dawson K, Mitchell J, et al (2006) The Addenbrooke's Cognitive Examination Revised (ACE-R): a brief cognitive test battery for dementia screening. International Journal of Geriatric Psychiatry 21: 1078-85.

Morris JL, Heyman A, Mohs RC, et al (1989) The consortium to establish a registry for Alzheimer's disease (CERAD). Part I. Clinical and neuropsychological assessment of Alzheimer's disease. Neurology 39 : $1159-65$.

National Collaborating Centre for Mental Health (2011) Dementia: Supporting People with Dementia and Their Carers in Health and Social Care (NICE Clinical Guideline 42). National Institute for Health and Clinical Excellence.

Nuechterlein KH, Green MF, Kern RS et al (2008) The MATRICS Consensus Cognitive Battery, Part 1: Test selection, reliability, and validity. American Journal of Psychiatry 165: 203-13.

Palmer BW, Bondi MW, Twamley EW, et al (2003) Are late-onset schizophrenia spectrum disorders neurodegenerative conditions? Annual rates of change on two dementia measures. Journal of Neuropsychiatry Clinical Psychiatry and Clinical Neurosciences 15: 45-52.

Powchik P, Davidson M, Haroutunian V, et al (1998) Postmortem studies in schizophrenia. Schizophrenia Bulletin 24: 325-41.

Purohit DP, Davidson M, Perl DP, et al (1993) Severe cognitive impairment in elderly schizophrenic patients: a clinicopathological study. Biological Psychiatry 33: 255-60

Purohit DP, Perl DP, Powchik P, et al (1998) Alzheimer's disease and related neurodegenerative diseases in elderly patients with schizophrenia: a postmortem study of 100 cases. Archives of General Psychiatry 55: 205-11

Raina P, Santaguida P, Ismaila A, et al (2008) Effectiveness of cholinesterase inhibitors and memantine for treating dementia: evidence review for a clinical practice guideline. Annals of Internal Medicine 148: 379-97

Religa D, Laudon H, Styczynska M, et al (2003) Amyloid beta pathology in Alzheimer's disease and schizophrenia. American Journal of Psychiatry 160: 867-72.

Royal College of Psychiatrists (2006) Services for Younger People with Alzheimer's Disease and Other Dementias (Council Report CR135). Royal College of Psychiatrists.

Silverman DHS, Small GW, Chang CY, et al (2001) Positron emission tomography in evaluation of dementia: regional brain metabolism and long-term clinical outcome. JAMA 286: 2120-7. 
Stip E, Sepehry AA, Chouinard S (2007) Add-on therapy with acetyl cholinesterase inhibitors for memory dysfunction in schizophrenia: a systematic quantitative review. Clinical Neuropharmacology 30: 218-29

Stryjer R, Strous RD, Bar F, et al (2003) Beneficial effect of donepezil augmentation for the management of comorbid schizophrenia and dementia. Clinical Neuropharmacology 26: 12-7.

Wechsler D (1981) Manual for the Wechsler Adult Intelligence Scale Revised. Psychological Corporation.

Willis, R, Chan J, Murray J, et al (2009) People with dementia and their family carers' satisfaction with a memory service: a qualitative evaluation generating quality indicators for dementia care. Journal of Mental Health 18: 26-37.

Winokur G, Pfohl B, Tsuang M (1987) A 40-year follow up of hebephrenic catatonic schizophrenia. In Schizophrenia and Aging (eds N Miller, G Cohen): 52-60. Guilford Press.

Xie J, Brayne C, Matthews FE, et al (2008) Survival times in people with dementia: analysis from population based cohort study with 14 year follow-up. BMJ 336: 258-62.

Zakzanis KK, Andrikopoulos J, Young D, et al (2003) Neuropsychological differentiation of late onset schizophrenia and dementia of the Alzheimer's type. Applied Neuropsychology 10: 105-14.

\section{MCOs}

Select the single best option for each question stem

\section{As regards schizophrenia:}

a cognitive impairment is a feature of schizophrenia

b cognitive impairment is a criterion used to diagnose schizophrenia in DSM-IV and ICD-10

c cognitive impairment always progresses in schizophrenia

d Alzheimer's disease invariably develops in older people with schizophrenia

e dementia in schizophrenia is accepted as a separate nosological entity.

2 Of the following, the least useful scale for the diagnosis of dementia with schizophrenia is:

a the MMSE

b the CERAD neuropsychological battery c the MATRICS battery

$d$ the ACE-R

e the ADAS-L Cog.

3 Radiological signs specific in diagnosing dementia in schizophrenia include:

a hippocampal atrophy

b ventricular enlargement

c white matter lesions

d temporal lobe atrophy

e none of the above.

4 Of the following, the least useful in improving cognition in comorbid dementia and schizophrenia is thought to be:

a first-generation antipsychotics

b second-generation antipsychotics

c galantamine

d rivastigmine

e donepezil
5 As regards the diagnosis of dementia in schizophrenia:

a cognitive impairment always means that dementia is present

b the presence of dementia in schizophrenia usually means that post-mortem examination will reveal Alzheimer's disease pathology

c functional imaging shows a clear diagnostic pattern

d the diagnosis can be made only after a history of cognitive and functional decline has been identified

e the diagnosis should only be made by an old age psychiatrist. 